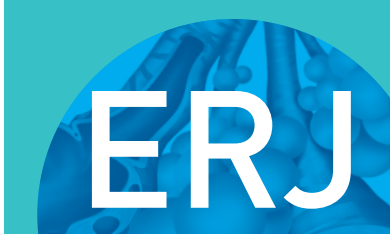

open research

\title{
RNA sequencing of transplant-stage idiopathic pulmonary fibrosis lung reveals unique pathway regulation
}

\author{
Pitchumani Sivakumar ${ }^{1}$, John Ryan Thompson², Ron Ammar², Mary Porteous ${ }^{3}$, \\ Carly McCoubrey ${ }^{3}$, Edward Cantu $1 \mathrm{II}^{4}$, Kandasamy Ravi ${ }^{5}$, Yan Zhang $^{5}$, Yi Luo ${ }^{6}$, \\ Denis Streltsov ${ }^{1}$, Michael F. Beers ${ }^{3,7}$, Gabor Jarai ${ }^{1}$ and Jason D. Christie ${ }^{3,7}$
}

Affiliations: ${ }^{1}$ Fibrosis Translational Research and Development, Bristol-Myers Squibb Research and Development, Princeton NJ, USA. ${ }^{2}$ Translational Bioinformatics, Bristol-Myers Squibb Research and Development, Princeton NJ, USA. ${ }^{3}$ Pulmonary and Critical Care Medicine, University of Pennsylvania, Philadelphia PA, USA. ${ }^{4}$ Surgery Dept, University of Pennsylvania, Philadelphia PA, USA. ${ }^{5}$ Integrated Genomics, Bristol-Myers Squibb Research and Development, Princeton NJ, USA. ${ }^{6}$ Clinical Biomarkers, Bristol-Myers Squibb Research and Development, Princeton NJ, USA. ${ }^{7}$ PENN Center for Pulmonary Biology, University of Pennsylvania, Philadelphia PA, USA.

Correspondence: Pitchumani Sivakumar, Fibrosis Translational Research, Bristol-Myers Squibb, 311 Pennington Rocky Hill Road, Pennington, NJ 08534, USA. E-mail: pitchumani.sivakumarabms.com

ABSTRACT Idiopathic pulmonary fibrosis (IPF), the scarring of lung parenchyma resulting in the loss of lung function, remains a fatal disease with a significant unmet medical need. Patients with severe IPF often develop acute exacerbations resulting in the rapid deterioration of lung function, requiring transplantation. Understanding the pathophysiological mechanisms contributing to IPF is key to develop novel therapeutic approaches for end-stage disease.

We report here RNA-sequencing analyses of lung tissues from a cohort of patients with transplant-stage IPF $(n=36)$, compared with acute lung injury (ALI) $(n=11)$ and nondisease controls $(n=19)$, that reveal a robust gene expression signature unique to end-stage IPF. In addition to extracellular matrix remodelling pathways, we identified pathways associated with T-cell infiltration/activation, tumour development, and cholesterol homeostasis, as well as novel alternatively spliced transcripts that are differentially regulated in the advanced IPF lung versus ALI or nondisease controls. Additionally, we show a subset of genes that are correlated with percent predicted forced vital capacity and could reflect disease severity.

Our results establish a robust transcriptomic fingerprint of an advanced IPF lung that is distinct from previously reported microarray signatures of moderate, stable or progressive IPF and identifies hitherto unknown candidate targets and pathways for therapeutic intervention in late-stage IPF as well as biomarkers to characterise disease progression and enable patient stratification.

@ERSpublications

An RNA-Seq-based transcriptomic fingerprint of severe IPF enriched in pathways of T-cell infiltration/activation, tumour development and cholesterol homeostasis highlights novel splice variants, candidate targets and biomarkers in advanced IPF http://bit.ly/2YbTOv8

Cite this article as: Sivakumar P, Thompson JR, Ammar R, et al. RNA sequencing of transplantstage idiopathic pulmonary fibrosis lung reveals unique pathway regulation. ERJ Open Res 2019; 5: 00117-2019 [https://doi.org/10.1183/23120541.00117-2019].

This article has supplementary material available from openres.ersjournals.com

Received: 13 May 2019 | Accepted after revision: 15 June 2019

Copyright $\odot$ ERS 2019. This article is open access and distributed under the terms of the Creative Commons Attribution Non-Commercial Licence 4.0. 


\section{Introduction}

Idiopathic pulmonary fibrosis (IPF) is a fatal disease of unknown aetiology characterised by the scarring of the lung parenchyma, resulting in the progressive loss of lung function and eventual death [1]. Although two recently approved medications for IPF (pirfenidone (Esbriet) and nintedanib (Ofev)) modestly reduce lung function decline in moderate IPF, they do not halt or reverse fibrosis, and do not significantly improve quality of life [2-4]. Lung transplant still remains the only option to prolong survival in patients with severe IPF [5]. Therapeutic approaches to IPF targeting numerous inflammatory and tissue remodelling pathways have consistently failed in the clinic, in part due to limited disease understanding, and lack of predictive diagnostic/prognostic biomarkers. Several studies in the past have utilised microarray profiling [6-9] and more recently single-cell RNA sequencing $[10,11]$ of IPF patient-derived lung tissue to identify genes and/or pathways differentially regulated in comparison with controls or patients with other lung diseases, providing signatures for disease classification. Peripheral blood profiling across small cohorts of patients with IPF has also identified potential biomarkers of disease [12-15]. Where available, gene/ protein expression profiles have been associated with clinical diagnosis, disease severity and measures of lung function [8]. While these studies have shed light on pathways that could contribute to early, stable or progressive IPF, our knowledge of the pathways and mechanisms that contribute to severe/end-stage IPF remains very limited. Importantly, therapies targeting pathways identified to be dysregulated in patients with early/stable/progressive IPF have not been effective in the treatment of advanced IPF. Patients with severe IPF often develop additional lung complications including acute exacerbations, lung cancer and rapid decline in lung function, requiring lung transplantation [16]. The diagnosis of IPF often occurs very late in the clinical course, when the disease has progressed significantly. Thus, understanding of molecular mechanisms in severe IPF could help develop targeted therapies and personalised medicine approaches for this deadly disease.

We hypothesised that the molecular signature of severe IPF would be different from that of ALI and healthy controls and therefore, could help differentiate and stratify patients with advanced disease and identify novel therapeutic targets and biomarkers. Accordingly, we performed RNA sequencing on lung tissues from a cohort of patients with severe IPF that underwent lung transplantation $(\mathrm{n}=36)$ and compared this with tissues from nondiseased controls $(n=19)$ and patients with clinical and pathological acute lung injury (ALI) ( $n=11)$. Furthermore, we used regression analyses to identify genes most strongly associated with lung function. Additionally, we identified alternative splicing of a large number of genes in advanced IPF. Using these complementary approaches, we established a robust transcriptomic fingerprint of severe IPF, revealed several key pathways (T-cell infiltration, immune response, host defence, cholesterol homeostasis and prostaglandin synthesis) that are differentially regulated and highlighted candidate biomarkers and targets as well as alternative isoform regulation. Our work therefore is important in expanding the knowledge of uniquely altered pathways that could lead to lung function decline in end-stage IPF and the identification of new biomarkers to predict organ failure and potential targets to treat advanced IPF.

\section{Materials and methods}

Human subjects and lung tissue acquisition

All human subject sample acquisitions (described in supplementary methods) and experiments were conducted with the appropriate approval from the Institutional Review Board (IRB 806468, IRB 813685).

TABLE 1 Profiles of control donor, idiopathic pulmonary fibrosis (IPF) and acute lung injury (ALI) patient cohorts used in the study

\begin{tabular}{lcccc} 
& IPF $(\mathbf{n}=\mathbf{3 6})$ & ALI $(\mathbf{n = 1 1 )}$ & Control (n=19) & p-value \\
\hline Male & $28(77.8 \%)$ & $5(45.5 \%)$ & $10(52.6 \%)$ & 0.056 \\
Age years & $64(60-66)^{*}$ & $43.5 \pm 16.9(n=10)$ & $33.2 \pm 25.6$ & 0.001 \\
Race & & & & 0.582 \\
$\quad$ White & $30(83.3 \%)$ & $10(90.9 \%)$ & $15(78.9 \%)$ & \\
African American & $3(8.3 \%)$ & $1(9.1 \%)$ & $3(10.5 \%)$ & \\
Asian & $3(8.3 \%)$ & 0 & 0 & \\
Other & 0 & 0 & 0 & $<0.001$ \\
Unknown & 0 & $3(27.3 \%)$ & $6(31.6 \%)$ & \\
Smoke exposure & $22(61.1 \%)$ & &
\end{tabular}

Data are presented as median (interquartile range) or mean \pm SD, unless otherwise stated. *: $p<0.05$ between IPF and control and IPF and ALI groups. 
The clinical profile and demographics of IPF, ALI and control subjects are listed in table 1. Details of the acquisition protocol are provided in the supplementary material.

\section{RNA sequencing}

Illumina TruSeq Stranded Total RNA Library Prep Kit with Ribo-Zero (cat. No RS-122-2203, Illumina Inc., San Diego, CA) was used to generate sequencing libraries per manufacturer's recommendation. Gene expression was determined via RNA-Seq libraries run on an Illumina HiSeq 25000 platform producing 75 bp paired-end (PE) reads. We generated on average 40 million PE reads for each sample. Reads were aligned to the human genome (GRch38) with the Omicsoft Sequence Aligner [17]. Gene and transcript abundance was determined using Ensembl release 90 human gene models [18] using RSEM [19]. All the gene expression data were deposited in the National Center for Biotechnology Information Gene Expression Omnibus (GSE 134692).

\section{RNA-Seq data analyses}

All RNA-Seq data were processed in $\mathrm{R}$ with the Bioconductor packages [20]. RNA-Seq samples were TMM (Trimmed Mean of M Values) normalised [21] with the edgeR package [22]. Outlier detection was performed using t-distributed stochastic neighbour embedding (t-SNE) [23]. Contributions of known variables (disease state, age, sex) to the data variance were assessed by principal variance component analyses. Differential gene expression contrasts between treatment groups was performed using the limma package [24]. Pathway enrichment was computed using Genego (Metacore) and MetaBase (Clarivate Analytics) version 6.34.69200. We also performed ensemble gene set enrichment analyses using the molecular signature database (MSigDB), a comprehensive database from the Broad Institute encompassing numerous curated public gene sets and pathways [25].

\section{Alternative isoform regulation}

We used JunctionSeq software for detecting the differential usage of exons and splice junctions from RNA-Seq data [26]. This enabled the determination of alternative isoform regulation. Testable loci identified through JunctionSeq were further filtered by excluding those with mean counts $\geqslant 10$ and an adjusted $\mathrm{p}$-value $\leqslant 0.05$.

\section{Statistical analyses}

Total gene expression data were analysed using a fold change/false discovery rate (FDR) cut-off of $2 \mathrm{X} /$ 0.001 (for comparing IPF and control) or 1.5X/0.1 (for comparing IPF versus control and ALI versus control signatures) to generate gene lists for pathway analyses. Individual gene expression was compared across groups by one-way ANOVA followed by Tukey's post-test with differences considered statistically significant at $\mathrm{p} \leqslant 0.05$.

a)

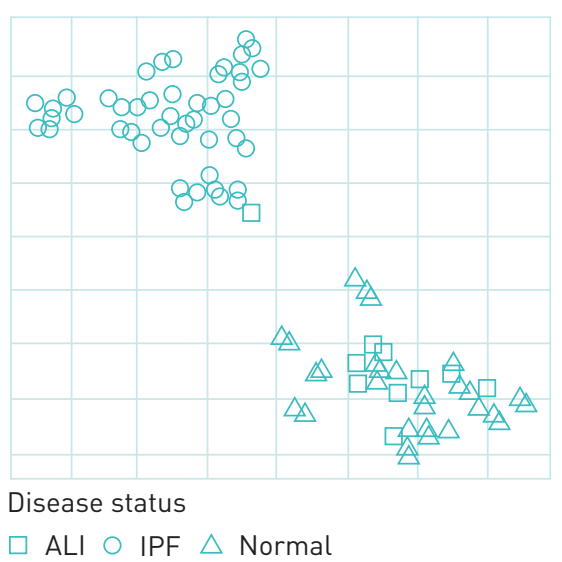

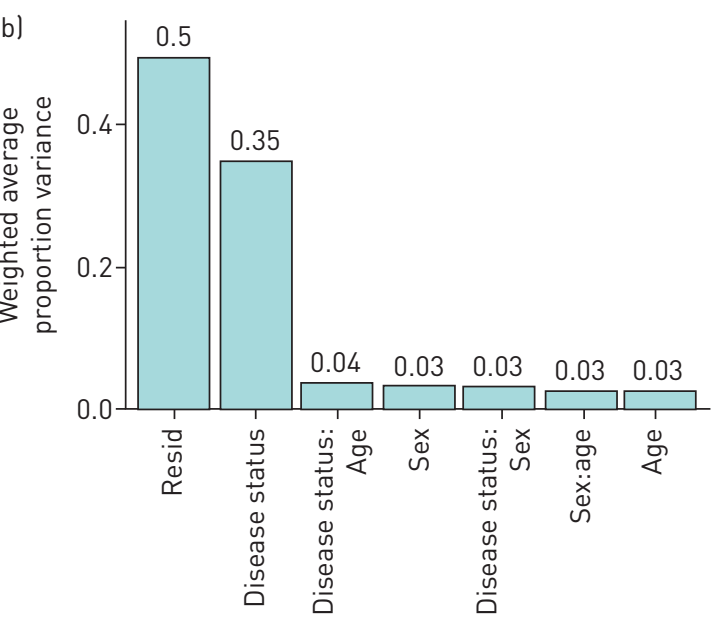

FIGURE 1 Quality control analyses of RNA-sequencing data from idiopathic pulmonary fibrosis (IPF) ( $n=36$ ), acute lung injury (ALI) $(n=10)$ and control $(n=20)$ lung tissue. a) Principal component analyses of batch-corrected normalised expression data using the t-scholastic neighbourhood enrichment (t-SNE method). b) Principal variance component analyses were used to assess the relative contribution of patient variables to variance in the normalised expression across the samples. 


\section{Results}

t-SNE variance analyses

We used the t-SNE method to visualise the distribution of the expression data across control and diseased samples and identify outliers. As shown in figure 1a, there was a clear separation of the IPF samples from both the control and ALI samples. To assess the contribution of donor variables to gene expression, we performed variance partition analyses. Figure $1 \mathrm{~b}$ shows that the largest contributor to the variance among known variables was the IPF or control disease state reaffirming that the difference in normalised gene expression was driven primarily by the IPF or control disease state ("Residual" represents a residual sum of unknown variables and could include polypharmacology, medications, and genetic factors among many others). Other known parameters such as age (although significantly higher in IPF), sex or ethnicity did not appear to significantly contribute towards the changes in expression.

\section{Differential gene expression and pathway regulation in IPF}

Figure 2a shows the heatmap of scaled gene expression of the samples generated by hierarchical clustering and represents a transcriptomic fingerprint of advanced IPF. Greater than $90 \%$ of the IPF samples clearly clustered together confirming that they represent a unique cohort of patients with a distinct molecular signature. Given the robust separation of the IPF samples from both ALI and control samples, we first analysed the IPF fingerprint through Metacore pathway analyses using a stringent cut-off of fold change $\geqslant 2$ and an adjusted p-value $\leqslant 0.001$. Consistent with an advanced disease state, we identified a strong upregulation of pathways associated with tumour cell infiltration and development of cancer (figure 3a) in addition to expected alterations in extracellular matrix (ECM) remodelling pathways. Notably, T-cell activation and survival pathways (CD4, CD8 and regulatory T-cells) were strongly upregulated concomitant with a robust increase in the expression of the checkpoint effectors programmed cell death (PD)1, LAG3 and CTLA4, the T-cell costimulatory receptor CD28 as well as chemokine/chemokine receptors including CCR5, CCR6, CXCR3 and CXCR5 (figure 3b). The T-cell signature in this cohort was more prominent than the classical or alternatively activated monocyte/macrophage signature. Of note, genes involved in inflammatory signalling leading to myeloid-derived suppressor cells or M2 macrophages were strongly downregulated (figure 3c; calgranulin/S100A8/S100A9, RAGE and TLR4, although TLR4 did not meet the stringent cut-off for this analysis).

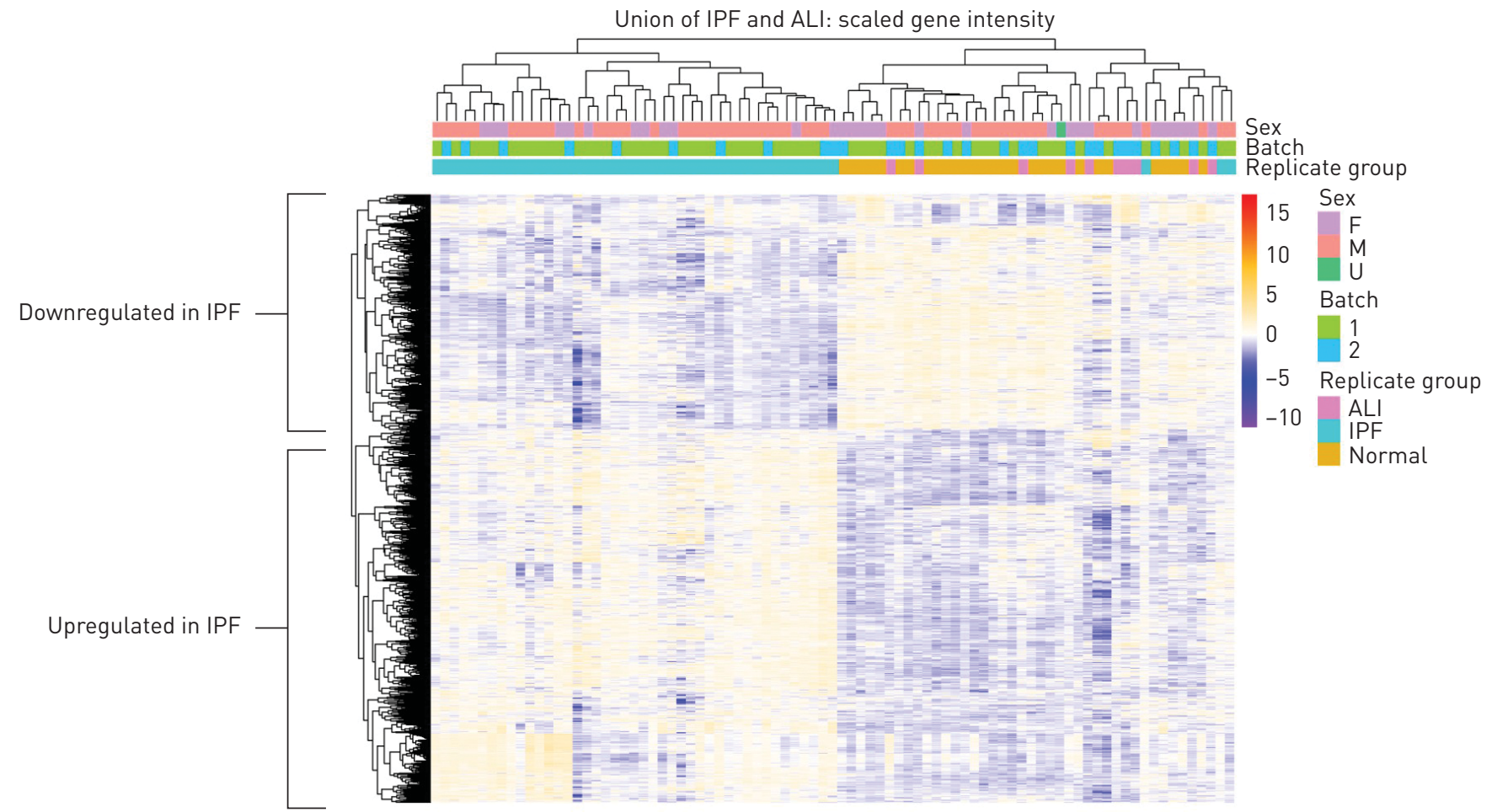

FIGURE 2 Transcriptomic fingerprint of advanced idiopathic pulmonary fibrosis (IPF) lung. Differential gene expression contrasts between treatment groups was performed using the limma package. Heatmap showing scaled intensity of individual genes in rows and samples in columns. Note samples predominantly cluster based on disease status. ALI: acute lung injury; F: female; M: male; U: unknown. 

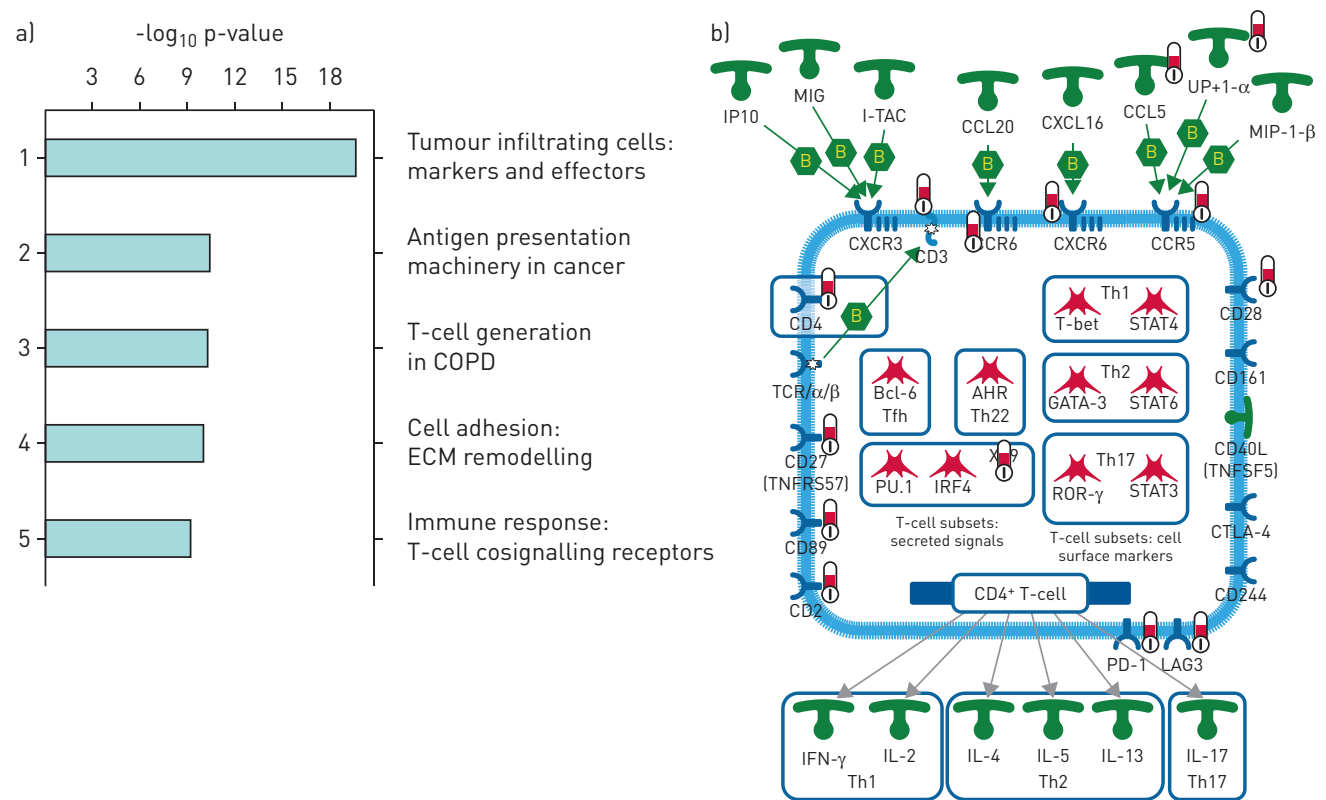

c)

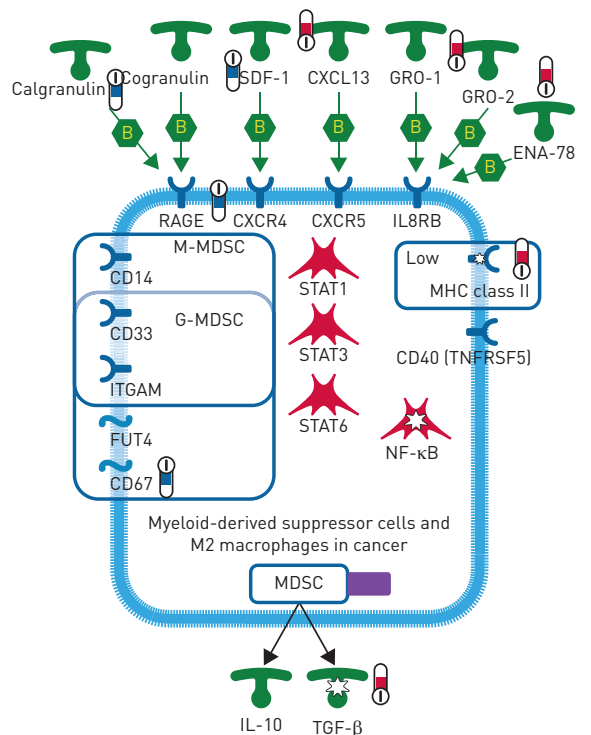

FIGURE 3 Differential pathway regulation in advanced idiopathic pulmonary fibrosis (IPF) lung. a) Metacore analyses of pathway enrichment in IPF versus control lungs using a filter of positive and negative fold change $\geqslant 2$ and adjusted $p$-value of $\leqslant 0.001$ b) Genego pathway map of tumour-infiltrating T-cells showing selected markers and key effector molecules. cl Genego pathway map of myeloid-derived suppressor cells in cancer. Red and blue thermometers indicate pathway components showing increased or decreased expression in IPF respectively. ECM: extracellular matrix; IP10: 10-kDa interferon- $\gamma$-inducible protein; MIG: monokine induced by interferon- $\gamma$; I-TAC: interferon-inducible T-cell $\alpha$-chemoattractant; MIP: macrophage inflammatory protein; Th: T-helper; STAT: signal transducer and activator of transcription; TCR: T-cell receptor; TNFR: tumour necrosis factor receptor; Tfh: T-follicular helper; IRF: interferon regulatory factor; ROR: RAR-related orphan receptor; CTLA: cytotoxic T-lymphocyte-associated protein; PD: programmed death receptor; LAG: lymphocyte activation gene product; IFN: interferon; IL: interleukin; SDF: stromal cell-derived factor; GRO: growth-related oncogene protein; ENA: epithelium-derived neutrophil-activating protein; RAGE: receptor of advanced glycosylation end-products; IL8R: interleukin-8 receptor; M-MDSC: monocytic myeloid-derived suppressor cell; G-MDSC: granulocytic myeloid-derived suppressor cell; ITGAM: integrin subunit $\alpha \mathrm{M}$. FUT: fucosyltransferase; MHC: major histocompatibility complex; TNFRSF: tumour necrosis factor receptor superfamily; NF- $\kappa B$ : nuclear factor, $\kappa$-light chain enhancer of activated B-cells; TGF: transforming growth factor. 
Comparison of pathway regulation in IPF and ALI patient cohorts

We employed multiple methods to identify the key genes and pathways that were uniquely regulated in the IPF and ALI patient cohorts. The complete list of gene changes for the different contrasts is provided in Supplementary Table T1. Figure 4 shows a contingency matrix of the number of genes that were significantly modulated across the different comparison groups with a fold change/adjusted p-value cut-off of 1.5/0.1. The IPF group compared with non-diseased controls expectedly showed the maximum number of gene changes, and genes commonly regulated in ALI and IPF mostly changed in the same direction. The volcano plots (figure 4b) further confirm that the number and magnitude of gene changes were far higher in the IPF versus control contrast. Genes uniquely regulated in each of these cohorts were analysed using the MetaBase pathway analyses tool. Immune response and T-cell activation pathways were strongly upregulated in IPF when compared with ALI, in addition to several expected fibrotic pathways including cell adhesion, ECM remodelling and epithelial mesenchymal transition (EMT) (figure 5a). Surprisingly, we also found that the cholesterol homeostasis pathway was strongly and significantly downregulated in IPF compared with both controls. In contrast, analyses of gene subsets unique to ALI revealed a strong regulation of cell cycle-associated pathways (Figure 5b). Importantly, within commonly regulated pathways across cohorts, we noted that a relatively greater fraction of genes within the "T-cell cosignalling pathway" and "Cell adhesion-remodelling" pathway were altered in IPF suggesting increased involvement of these genes/pathways in advanced IPF. Further analyses of the data with the Msigdb tool confirmed many of these findings (supplementary figure S1) and additionally identified a subset of robustly downregulated genes within the cholesterol homeostasis pathway overlapping with statin-regulated genes in the human lung (supplementary figure S2).

Since the patients in the IPF cohort in this study were significantly older compared with controls or the ALI cohort, we performed the analyses with the inclusion of age in the statistical model (see supplementary material figures S3 and S4); however, such inclusion did not significantly change the findings with respect to variance or pathway analyses.

\section{Alternative isoform regulation in IPF tissue}

An important advantage of using RNA-Seq is the ability to detect gene splicing, which is not as accessible with microarray platforms. Accordingly, we analysed the gene expression data using the "JunctionSeq" tool to identify splice variants and differentially regulated exons to provide a measure of alternative isoform regulation. After applying count threshold and FDR filters, we identified 2723 exon junctions that were differentially regulated in IPF lung tissue. The top 15 loci showing the strongest isoform regulation are shown in figure 6a (the full list of differentially regulated loci is available in supplementary table T2). A Metacore analysis of genes with significantly altered exon junctions revealed several fibrosis-relevant pathways including cytoskeleton remodelling, cell adhesion, transforming growth factor (TGF)- $\beta$ and vascular endothelial growth factor (VEGF) signalling. Interestingly, pathways involved in lysophosphatidic acid (LPA)-mediated G protein-coupled receptor signalling were also enriched (figure $6 \mathrm{~b}$ ). In comparison with the only RNA-Seq data published previously with a small cohort of IPF lung tissue ( $\mathrm{n}=8$, Nance et al., 2014 [27]), we identified a three-fold greater number of significantly regulated loci with 83 genes identified in common across these two studies (figure 7a). Importantly, several of the common genes are implicated in or associated with fibrosis. Wnt2b, a common gene in both our study and that of Nance et al., appeared to show a significantly altered expression of multiple exons, while the overall expression of

a)
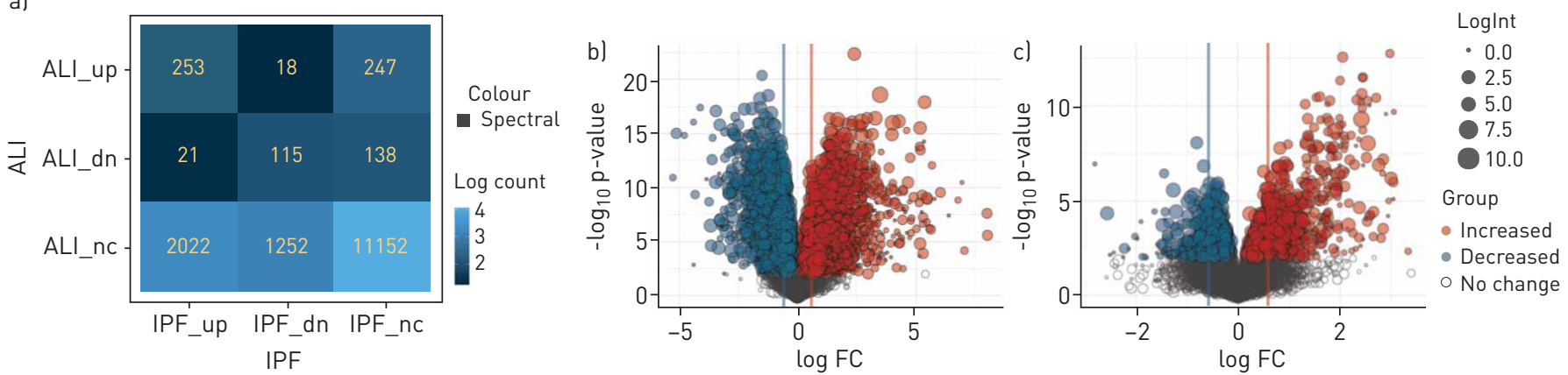

FIGURE 4 Comparison of gene expression changes in idiopathic pulmonary fibrosis (IPF) versus control and acute lung injury (ALI) versus control contrasts. Differential gene expression contrasts between treatment groups was performed using the limma package. Gene lists were generated using a cut-off of positive and negative fold change $\geqslant 1.5$ and adjusted $p$-value of $\leqslant 0.1$. a) Contingency table shows numbers and direction of movement of differentially altered genes and a measure of overlap/divergence between contrast groups. b) and c) Volcano plots showing the number and magnitude of significant gene changes across the IPF versus control and ALI versus control contrasts, respectively. 
a)

Cholesterol biosynthesis

Immune response: T-cell cosignalling

Cell adhesion: ECM remodelling

Immune response: T-cell subsets

Colorectal cancer

Immune response: IL-4 type 2 immunity

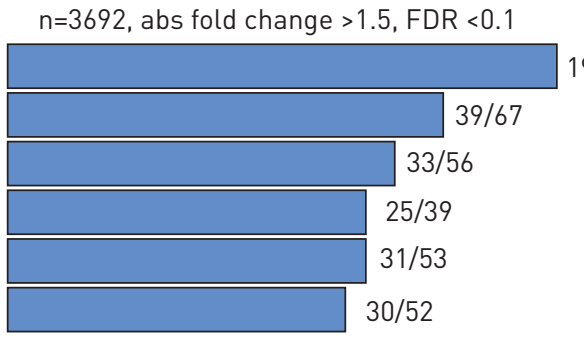

$19 / 21$

b)

Cell cycle: metaphase checkpoint

Cell cycle: APC regulation of

Cell cycle: spindle assembly

Cell cycle: chromosome condensation

DNA damage: ATM/ATR: G2/M checkpoint

Cell adhesion: ECM remodelling $\mathrm{n}=611$, abs fold change $>1.5$, FDR $<0.1$

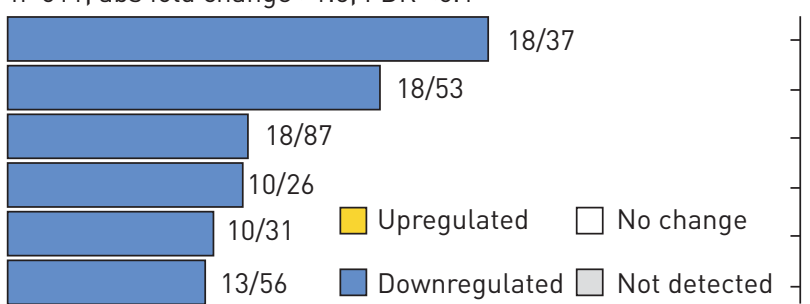

Fraction of genes

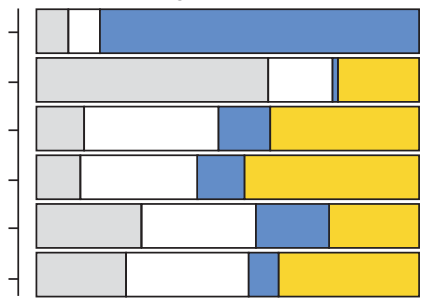

Fraction of genes

FIGURE 5 Comparison of pathway enrichment in a) idiopathic pulmonary fibrosis (IPF) and b) acute lung injury (ALI) cohorts. Gene lists were generated using a cut-off of positive and negative fold change $\geqslant 1.5$ and adjusted $p$-value of $\leqslant 0.1$. Left panels: pathways uniquely enriched in the IPF or ALI cohort. Numbers against each bar represent number of differentially altered genes compared with the total number of genes defining that pathway. Right panels: fraction of genes within the pathway that show increase, decrease or no alteration. ECM: extracellular matrix; FDR: false discovery rate.

the gene was unchanged (figure $7 \mathrm{~b}$ ). Moreover, the altered exonic expression appears to map to a specific transcript, suggesting this transcript is expressed differentially in patients with IPF.

\section{Association between gene expression and lung function}

We correlated gene expression to \% predicted forced vital capacity (FVC), as a marker of disease severity, using Spearman's correlation analyses for nonlinear association studies. We identified nearly 300 genes that were significantly correlated with \% predicted FVC (supplementary table T3). The top 15 genes that correlated positively with a decline in lung function are shown in figure 8a, and a Metacore pathway analyses that indicated that the gene subsets were enriched in fibrosis-relevant pathways are shown in figure $8 \mathrm{~b}$. The correlation of 2 of the top 15 genes (secretogranin-2 and semaphorin-3C) presented in figure $8 \mathrm{c}-\mathrm{e}$ demonstrates that their expression in IPF tissue was also significantly elevated.

a)

\begin{tabular}{|c|c|c|}
\hline Gene name & $\begin{array}{l}\text { Exon or } \\
\text { junction ID }\end{array}$ & Gene description \\
\hline HOPX & E014 & HOP homeobox \\
\hline HOPX & J037 & HOP homeobox \\
\hline MYLK & E014 & Myosin light chain kinase \\
\hline MYLK & E013 & Myosin lght chain kinase \\
\hline HOPX & E013 & HOP homeobox \\
\hline MYLK & E015 & Myosin light chain kinase \\
\hline EPB41L2 & E028 & Erythrocyte membrane protein band 4.1 \\
\hline ARMC9 & E028 & Armadillo repeat containing 9 \\
\hline EPB41L2 & $\mathrm{J} 105$ & Erythrocyte membrane protein band 4.1 \\
\hline ARMC9 & E023 & Armadillo repeat containing 9 \\
\hline WINT2B & E001 & Wnt family member $2 \mathrm{~B}$ \\
\hline WNT2B & E002 & Wnt family member $2 \mathrm{~B}$ \\
\hline HOPX & E015 & HOP Homeobox \\
\hline
\end{tabular}

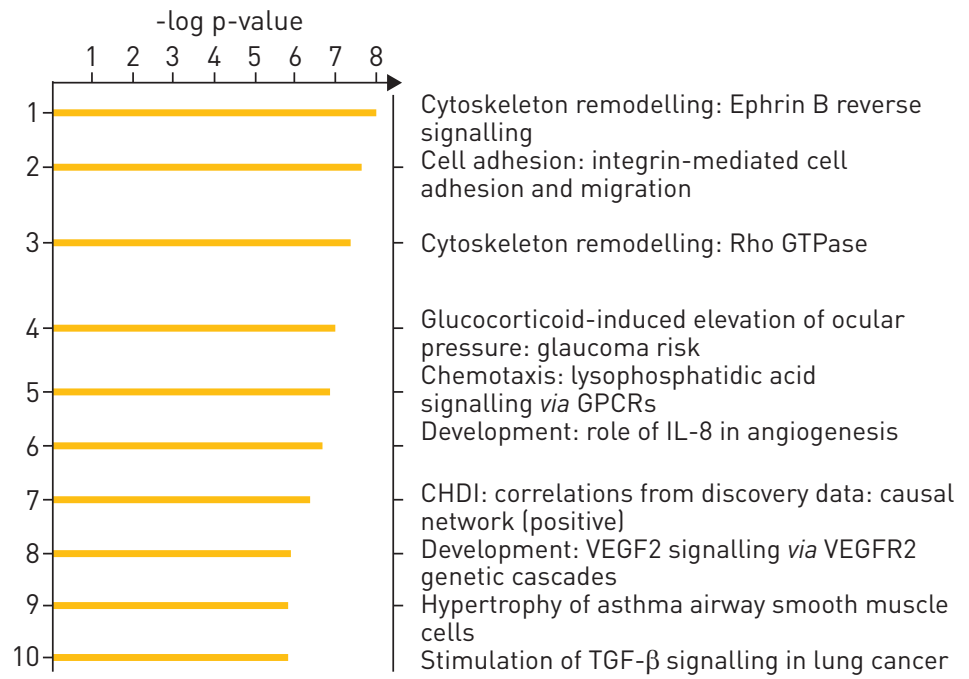

FIGURE 6 Alternative isoform regulation in advanced idiopathic pulmonary fibrosis (IPF) lung. a) List of top loci that show alternative splicing in the IPF lung analysed with the 'JunctionSeq' tool. b) Metacore analyses of pathways enriched by genes that show significant alternative isoform/ exon usage in the IPF lung ladjusted $p$-value $\leqslant 0.05$ ). GPCR: G-protein coupled receptor; IL: interleukin; VEGF: vascular endothelial growth factor; VEGFR: vascular endothelial growth factor receptor; TGF: transforming growth factor. 
a)
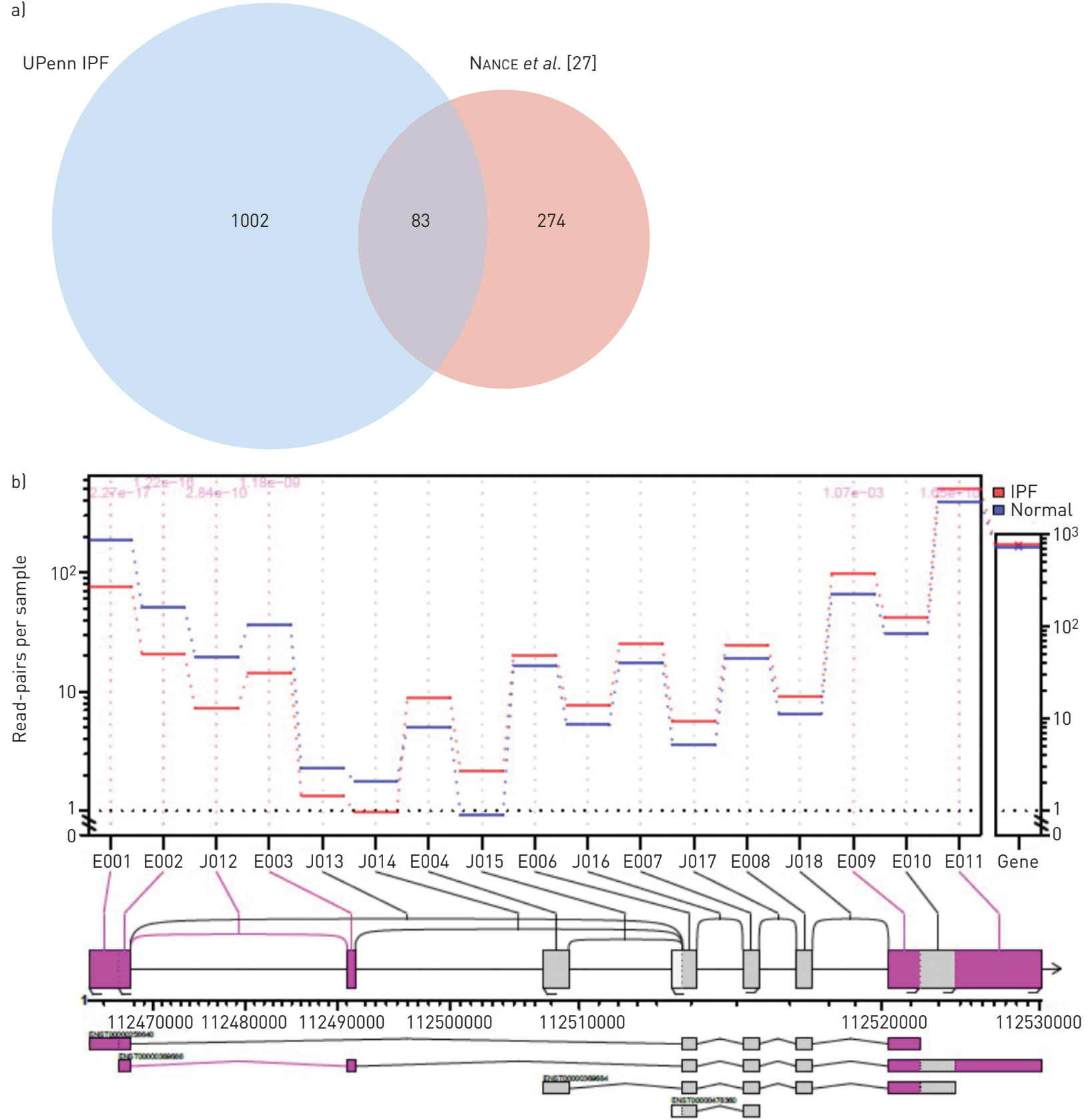

FIGURE 7 a) Comparison of alternative transcripts identified in idiopathic pulmonary fibrosis (IPF) lung in our study (Upenn IPF) versus those identified in a published study by Nance et al. (2014). b) Alternative isoform usage in Wnt2b gene. Note the higher expression of certain exons and lower expression of others in the IPF lung.

\section{Discussion}

We present here a markedly distinct transcriptomic fingerprint of advanced IPF obtained through RNA-sequencing analysis of a cohort of patients with transplant-stage IPF in comparison with nondiseased controls and patients with ALI. In addition to previously reported pathways involved in epithelial injury and ECM homeostasis, our study identified several novel pathways and candidate genes that are altered in end-stage disease, including T-cell infiltration/activation pathways, cell-matrix interactions, cholesterol homeostasis and steroid biosynthesis. We also demonstrate extensive alternative transcript/ exon/isoform usage in IPF, identifying novel splice variants that may be involved in disease pathology. Additionally, we show a novel subset of genes whose expression appears to be correlated with lung function decline in advanced IPF. 
a)

\begin{tabular}{lccc}
\hline Gene symbol & Description & Correl. coeff. & p-value \\
\hline SLC44A1 & Solute carrier family 44 member 1 & -0.62479102 & $6.02081 \times 10^{-5}$ \\
SCG2 & Secretogranin II & -0.588154207 & 0.000202637 \\
GL01 & Glyoxalase I & -0.532567317 & 0.000989844 \\
USP53 & Ubiquitin-specific peptidase 53 & -0.528075448 & 0.001112177 \\
TPST2 & Tyrosylprotein sulfotransferase 2 & -0.522179868 & 0.001292849 \\
VGLL3 & Vestigial-like family member 3 & -0.518109111 & 0.001432208 \\
XPOT & Exportin for tRNA & -0.514459467 & 0.001568197 \\
PI5 & Peptidase inhibitor 15 & -0.511090565 & 0.001703649 \\
PTPRG & Protein tyrosine phosphatase, receptor type G & -0.510809823 & 0.001715386 \\
ERFE & Erythroferrone & -0.503370163 & 0.002053369 \\
SACS & Sacsin molecular chaperone & -0.494947907 & 0.002504995 \\
BAG2 & BCL2-associated athanogene 2 & -0.48175304 & 0.003386762 \\
KIAA1324L & KIAA1324 like & -0.480349331 & 0.003494772 \\
TSPAN5 & Tetraspanin 5 & -0.479366734 & 0.003572144 \\
SEMA3C & Semaphorin 3C & -0.477822654 & 0.003696731 \\
\hline
\end{tabular}

b)
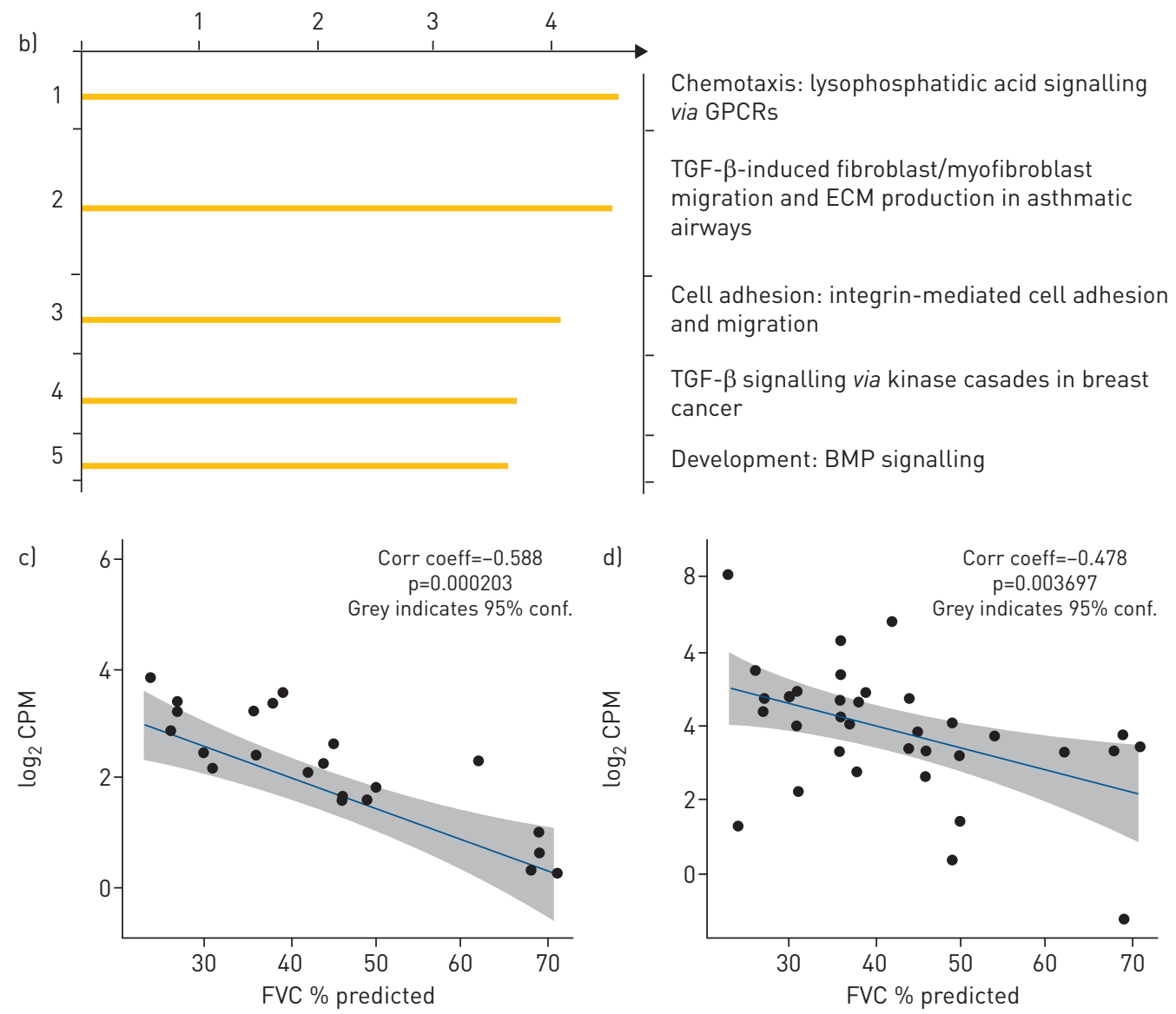

e)

\begin{tabular}{lcc}
\hline Gene & $\begin{array}{c}\text { log fold change } \\
\text { (IPF versus healthy) }\end{array}$ & Adjusted p-value \\
\hline SCG2 & 1.740 & 0.0000827063 \\
SEMA3C & 0.934 & 0.0000013766 \\
\hline
\end{tabular}

FIGURE 8 Correlation of idiopathic pulmonary fibrosis (IPF) lung gene expression to lung function. a) List of top genes that show significant inverse correlation to \% predicted forced vital capacity (FVC) as assessed by Spearmen's correlation analyses. b) Metacore analyses of pathways enriched by genes that show significant correlation with \% predicted FVC (adjusted p-value $\leqslant 0.05$ ). c) and d) Correlation plots of gene expression of SCG2 and SEMA3C, two candidate genes identified through Spearman's analyses as significantly correlated with \% predicted FVC. e) Gene expression intensity of SCG2 and SEMA3C confirming significant increase of these genes in IPF lung compared with control lung. tRNA: transfer RNA; GPCR: G-protein coupled receptor; TGF: transforming growth factor; ECM: extracellular matrix; BMP: bone morphogenetic protein; FVC: forced vital capacity. 
The molecular signature of our IPF cohort is distinctly different from past studies that have used lung tissues from patients with IPF with mild-to-moderate disease. Yang et al. [9] investigated the transcriptomic profile of IPF/usual interstitial pneumonia from the Lung Tissue Research Consortium and identified subgroups of patients with alterations in cilium-associated genes and epithelial injury pathways, further validating their findings with samples from the National Jewish Health cohort. Notably, the mean $\% \mathrm{FVC}$ in their test cohort was $\geqslant 59 \%$. In contrast, the mean \% FVC of patients in our study was $44 \%$, with a majority of patients at $\leqslant 30 \%$. Likewise, microarray studies by Depianto et al., with a cohort of 40 patients with IPF, identified two primary clusters of coregulated genes representing bronchiolar epithelium and lymphoid aggregates [28]. Worsening IPF pathology remains a significant risk factor for acute exacerbations, development of lung cancer and additional lung complications that warrant a lung transplant. Konishi et al. [6] showed that the gene expression profile of acute exacerbations of IPF (AE-IPF) was mostly similar to that of stable IPF with only a few genes significantly altered between the two groups. Interestingly, these authors also observed a downregulation of AGER (receptor for advanced glycation end products) as we noted in our cohort of advanced IPF. However, defensin a3 (DEFA3) was strongly reduced in our cohort in contrast with Konishi's study as well as to a separate study by Yang et al. [14] on the peripheral blood transcriptome of patients with IPF that identified DEFA3 to be increased in progressive disease. The apparent differences may be due to the different patient profiles and measures of disease severity. The AE-IPF cohort in Konishi's study had a mean \% FVC of 55\% and a lower DLCO (diffusing capacity of the lung for carbon monoxide) of 36\%, and Yang's study used DLCO as the disease classifier.

Consistent with advanced disease, pathways involved in T-cell activation and cancer/tumour development were strongly enriched in the IPF molecular signature in our study. Notably, several checkpoint effector molecules including PD1 receptor, LAG3 and CTLA4 were shown to be upregulated in IPF tissue, indicating the possibility of immune exhaustion in end-stage IPF. The role of T-lymphocytes in human IPF and animal models of fibrosis has been recognised before [29]. Abnormal PD1 levels have been identified in human IPF, and human mesenchymal stem cell therapy was protective in a humanised mouse model of fibrosis through suppression of $\mathrm{CD}^{+} \mathrm{T}$-cell infiltration and activation by a PD1/programmed death ligand (PD-L) 1 axis [30]. It was recently reported that the increased expression of PD1 on CD4 ${ }^{+}$ T-cells in IPF lung and plasma promotes a T helper (Th)-17 mediated response and induces active TGF- $\beta$ secretion from stimulated T-cells [31]. Interestingly, the authors also show that knockout of PD1 as well as blockade of PD-L1 signalling ameliorates the fibrotic responses induced by bleomycin in mice. Our data emphasise the role of T-cell activation in advanced IPF and highlight the potential utility of this pathway as a disease classifier as well as a candidate therapeutic target. However, while the T-cell phenotype and lineage appear to be critically important in the context of IPF and particularly in severe IPF, more work is needed to understand their relative contribution to disease pathology.

While we noted increases in T-cell activation pathways, we also observed a reduction in genes involved in the activation of myeloid-derived suppressor cells and M2 macrophages (calgranulin, AGER and TLR4). Myeloid-derived suppressor cells are elevated in the peripheral blood of patients with IPF and inversely correlate with lung function [32]. Alternatively activated (M2) macrophages, described to be abundant in both murine and human fibrosis are known to drive anti-inflammatory and profibrotic responses simultaneously [33]. These macrophages also produce chemokines that recruit T-lymphocytes into the injured lung. Our observation of reduction in signals that activate M2 differentiation, together with an upregulation of chemokines involved in T-cell recruitment may imply that T-cell-mediated pathways contribute to late-stage disease.

Biopsies of early IPF are not in standard clinical practice and would be very difficult to procure in sufficient numbers to include in the study. Therefore, in our study design, we specifically included ALI as an additional comparator because both IPF and ALI represent severe lung pathologies, albeit with different aetiologies. Only cell adhesion and ECM remodelling pathways were commonly regulated in both these cohorts in comparison with non-disease controls. The IPF signature was rich in T-cell/immune response while the ALI signature was enriched in genes/pathways involved in cell cycle regulation. Thus, the IPF signature that we present here could be uniquely representative of end-stage lung fibrosis. Intriguingly, the cholesterol homeostasis pathway was significantly downregulated in our patients with advanced IPF with the signature overlapping with published statin-responsive genes in the lung [34], a finding that should be further explored and perhaps validated in independent cohorts. In addition, genes within lipoprotein steroid and fatty acid biosynthesis pathways were also strongly under-expressed in the IPF cohort. Given that lipid-lowering therapy and reduced lipoprotein levels have been shown to be profibrotic in preclinical and clinical studies $[35,36]$, our data lend further credence to the hypothesis that lipoprotein levels are inversely correlated with the pathogenesis of interstitial lung disease, although the mechanisms contributing to this effect remain to be investigated. 
To date, there is only one study investigating alternative splicing in the IPF lung obtained through RNA sequencing of a small cohort of eight lungs from patients with IPF [27]. In that study, the authors used DEXseq to demonstrate alternative exon usage in known fibrosis-related genes including periostin and collagen 6. Our study identifies, for the first time, a larger subset of genes that exhibit alternative transcript/isoform usage in advanced IPF, opening up hitherto unknown targets and pathways for therapeutic intervention or as biomarkers of disease severity. Top pathways enriched in differentially spliced genes were found to be involved in fibrotic signalling (TGF, VEGF, ephrin B and LPA pathways) and cytoskeletal/ECM remodelling. Interestingly, we found HOPX (homeobox protein $\mathrm{x}$ ), a known marker of alveolar Type I cells [37] as the top alternatively spliced gene in our study. A recent study showed that HOPX impacts alveolar epithelial injury and fibrosis and is decreased in human IPF tissue [38] contributing to lung function decline potentially through a failure of epithelial cell regeneration. Our expression data (downregulation of HOPX in IPF versus control) confirm these findings and suggest that alternative splicing of HOPX could play a role in the development of fibrosis.

We note that previously reported biomarkers (MMP7) and genes implicated in alveolar epithelial cell (DIO2) and surfactant functions (SP-A, SP-C) and lineage-negative lung progenitor cell expansion (KRT5) changed in total expression, validating previous findings [12, 39, 40]. However, exploring differential expression of exons can help identify genes that may not change in total expression but could be alternatively spliced in disease, a mechanism that is likely to be missed with microarray studies. For example, we identified Wnt2b, a molecule that is antifibrotic in hepatic stellate cells [41] as a major alternatively spliced gene although the overall expression was not changed in IPF tissue.

Prognostic and/or predictive biomarkers of disease progression in IPF are critical for the early diagnosis and treatment of the disease. In our cohort, we investigated the correlation of gene expression with clinical measures of disease severity, using \% predicted FVC (recorded prior to transplantation) as the measure of lung function. Through Spearman's correlation analyses, we identified several genes whose expression was positively or negatively correlated with \% predicted FVC. Many of the identified associations are novel. Interestingly, many do not appear to significantly change when comparing overall expression, but their expression level increased or decreased with disease severity. Our studies thus may have identified novel biomarkers of disease severity that can be validated in other independent cohorts.

In summary, we have described for the first time an RNA-Seq-based transcriptomic fingerprint of severe IPF and a molecular signature that can be further evaluated as a potential disease classifier. We chose not to directly compare this signature with previously described signatures from microarray platforms, as there are significant differences in patient disease profiles and the methods used to derive the molecular signatures. However, we note that as opposed to previous studies that have largely described epithelial injury, cell-matrix remodelling pathways, our cohort of patients with advanced IPF exemplify other unique pathways that could contribute to the disease and potentially present interesting targets and biomarkers. Recently, in an approach that complements ours, Reyfman et al. [11] used single-cell sequencing of advanced IPF lungs to generate a single-cell atlas of IPF and identify distinct subpopulations of alveolar macrophages and epithelial cells that could drive fibrosis. Similar to our observations, their work also identifies immune response, ECM organisation and Wnt signalling pathways as regulated in advanced fibrosis. Although we did not use a single-cell approach, which would have been cumbersome given the large number of samples involved, we believe that the whole-organ signature of advanced IPF is important and informative, since the disease phenotype in IPF is a collective consequence of the heterogeneity in cell types within the organ. RNA-Seq coupled with single-cell analyses could provide a new step forward in the elusive quest for biomarkers and targets for the diagnosis and treatment of IPF.

Conflict of interest: P. Sivakumar has nothing to disclose. J.R. Thompson has nothing to disclose. R. Ammar has nothing to disclose. M. Porteous has nothing to disclose. C. McCoubrey has nothing to disclose. E. Cantu III has nothing to disclose. K. Ravi has nothing to disclose. Y. Zhang has nothing to disclose. Y. Luo has nothing to disclose. D. Streltsov has nothing to disclose. M.F. Beers reports grants from Bristol-Myers Squibb during the conduct of the study. G. Jarai has nothing to disclose. J.D. Christie reports grants from the NIH and Bristol-Myers Squibb during the conduct of the study and grants from the NIH, GlaxoSmithKline and Bristol-Myers Squibb outside the submitted work.

\section{References}

1 Martinez FJ, Collard HR, Pardo A, et al. Idiopathic pulmonary fibrosis. Nat Rev Dis Primers 2017; 3: 17074.

2 George PM, Wells AU. Pirfenidone for the treatment of idiopathic pulmonary fibrosis. Expert Rev Clin Pharmacol 2017; 10: 483-491.

3 Rogliani P, Calzetta L, Cavalli F, et al. Pirfenidone, nintedanib and N-acetylcysteine for the treatment of idiopathic pulmonary fibrosis: A systematic review and meta-analysis. Pulm Pharmacol Ther 2016; 40: 95-103.

4 Tomioka H, Takada H. Treatment with nintedanib for acute exacerbation of idiopathic pulmonary fibrosis. Respirol Case Rep 2017; 5: e00215. 
5 Caminati A, Cassandro R, Torre O, et al. Severe idiopathic pulmonary fibrosis: what can be done? Eur Respir Rev 2017; 26: 170047.

6 Konishi K, Gibson KF, Lindell KO, et al. Gene expression profiles of acute exacerbations of idiopathic pulmonary fibrosis. Am J Respir Crit Care Med 2009; 180: 167-175.

7 Kusko RL, Brothers JF 2nd, Tedrow J, et al. Integrated genomics reveals convergent transcriptomic networks underlying chronic obstructive pulmonary disease and idiopathic pulmonary fibrosis. Am J Respir Crit Care Med 2016; 194: 948-960.

8 Steele MP, Luna LG, Coldren CD, et al. Relationship between gene expression and lung function in idiopathic interstitial pneumonias. BMC Genomics 2015; 16: 869.

9 Yang IV, Coldren CD, Leach SM, et al. Expression of cilium-associated genes defines novel molecular subtypes of idiopathic pulmonary fibrosis. Thorax 2013; 68: 1114-1121.

$10 \mathrm{Xu} \mathrm{Y,} \mathrm{Mizuno} \mathrm{T,} \mathrm{Sridharan} \mathrm{A,} \mathrm{et} \mathrm{al.} \mathrm{Single-cell} \mathrm{RNA} \mathrm{sequencing} \mathrm{identifies} \mathrm{diverse} \mathrm{roles} \mathrm{of} \mathrm{epithelial} \mathrm{cells} \mathrm{in}$ idiopathic pulmonary fibrosis. JCI Insight 2016; 1: e90558.

11 Reyfman PA, Walter JM, Joshi N, et al. Single-cell transcriptomic analysis of human lung provides insights into the pathobiology of pulmonary fibrosis. Am J Respir Crit Care Med 2018; 198: 440-446.

12 Bauer Y, White ES, de Bernard S, et al. MMP-7 is a predictive biomarker of disease progression in patients with idiopathic pulmonary fibrosis. ERJ Open Res 2017; 3: 00074-2016.

13 Rosas IO, Richards TJ, Konishi K, et al. MMP1 and MMP7 as potential peripheral blood biomarkers in idiopathic pulmonary fibrosis. PLoS Med 2008; 5: e93.

14 Yang IV, Luna LG, Cotter J, et al. The peripheral blood transcriptome identifies the presence and extent of disease in idiopathic pulmonary fibrosis. PLoS One 2012; 7: e37708.

15 Herazo-Maya JD, Sun J, Molyneaux PL, et al. Validation of a 52-gene risk profile for outcome prediction in patients with idiopathic pulmonary fibrosis: an international, multicentre, cohort study. Lancet Respir Med 2017; 5: 857-868.

16 Lipsi R, Mazzola D, Caminati A, et al. Severe idiopathic pulmonary fibrosis: A clinical approach. Eur J Intern Med 2018; 50: 20-27.

$17 \mathrm{Hu}$ J, Ge H, Newman M, et al. OSA: a fast and accurate alignment tool for RNA-Seq. Bioinformatics 2012; 28: 1933-1934.

18 Zerbino DR, Achuthan P, Akanni W, et al. Ensembl 2018. Nucleic Acids Res 2018; 46: D754-D761.

19 Li B, Ruotti V, Stewart RM, et al. RNA-Seq gene expression estimation with read mapping uncertainty. Bioinformatics 2010; 26: 493-500.

20 Huber W, Carey VJ, Gentleman R, et al. Orchestrating high-throughput genomic analysis with Bioconductor. Nat Methods 2015; 12: 115-121.

21 Robinson MD, Oshlack A. A scaling normalization method for differential expression analysis of RNA-Seq data Genome Biol 2010; 11: R25.

22 Robinson MD, McCarthy DJ, Smyth GK. edgeR: a Bioconductor package for differential expression analysis of digital gene expression data. Bioinformatics 2010; 26: 139-140.

23 Van der Maaten L, Hinton G. Visualizing data using t-SNE. J Mach Learn Res 2008; 9: $2579-2605$.

24 Ritchie ME, Phipson B, Wu D, et al. Smyth GK. limma powers differential expression analyses for RNA-sequencing and microarray studies. Nucleic Acids Res 2015; 43: e47.

25 Subramanian A, Tamayo P, Mootha VK, et al. Gene set enrichment analysis: a knowledge-based approach for interpreting genome-wide expression profiles. Proc Natl Acad Sci USA 2005; 102: 15545-15550.

26 Hartley SW, Mullikin JC. Detection and visualization of differential splicing in RNA-Seq data with JunctionSeq Nucleic Acids Res 2016; 44: e127.

27 Nance T, Smith KS, Anaya V, et al. Transcriptome analysis reveals differential splicing events in IPF lung tissue PLoS One 2014; 9: e97550.

28 DePianto DJ, Chandriani S, Abbas AR, et al. Heterogeneous gene expression signatures correspond to distinct lung pathologies and biomarkers of disease severity in idiopathic pulmonary fibrosis. Thorax 2015; 70: 48-56.

29 Luzina IG, Todd NW, Iacono AT, et al. Roles of T lymphocytes in pulmonary fibrosis. J Leukoc Biol 2008; 83: 237-244

$30 \mathrm{Ni}$ K, Liu M, Zheng J, et al. PD-1/PD-L1 pathway mediates the alleviation of pulmonary fibrosis by human mesenchymal stem cells in humanized mice. Am J Respir Cell Mol Biol 2018; 58: 684-695.

31 Celada LJ, Kropski JA, Herazo-Maya JD, et al. PD-1 up-regulation on CD4( $\left.{ }^{+}\right) \mathrm{T}$ cells promotes pulmonary fibrosis through STAT3-mediated IL-17A and TGF- $\beta 1$ production. Sci Transl Med 2018; 10: eaar8356.

32 Fernandez IE, Greiffo FR, Frankenberger M, et al. Peripheral blood myeloid-derived suppressor cells reflect disease status in idiopathic pulmonary fibrosis. Eur Respir J 2016; 48: 1171-1183.

33 Braga TT, Agudelo JS, Camara NO. Macrophages during the fibrotic process: M2 as friend and foe. Front Immunol 2015; 6: 602 .

34 Lane J, van Eeden SF, Obeidat M, et al. Impact of statins on gene expression in human lung tissues. PLoS One 2015; 10: e0142037.

35 Podolanczuk AJ, Raghu G, Tsai MY, et al. Cholesterol, lipoproteins and subclinical interstitial lung disease: the MESA study. Thorax 2017; 72: 472-474.

$36 \mathrm{Xu}$ JF, Washko GR, Nakahira K, et al. Statins and pulmonary fibrosis: the potential role of NLRP3 inflammasome activation. Am J Respir Crit Care Med 2012; 185: 547-556.

37 Liebler JM, Marconett CN, Juul N, et al. Combinations of differentiation markers distinguish subpopulations of alveolar epithelial cells in adult lung. Am J Physiol Lung Cell Mol Physiol 2016; 310: L114-L120.

38 Ota C, Ng-Blichfeldt JP, Korfei M, et al. Dynamic expression of HOPX in alveolar epithelial cells reflects injury and repair during the progression of pulmonary fibrosis. Sci Rep 2018; 8: 12983.

39 Kinder BW, Brown KK, McCormack FX $\mathrm{I} \times \mathrm{JH}$, et al. Serum surfactant protein-A is a strong predictor of early mortality in idiopathic pulmonary fibrosis. Chest 2009; 135: 1557-1563.

40 Yu G, Tzouvelekis A, Wang R, et al. Thyroid hormone inhibits lung fibrosis in mice by improving epithelial mitochondrial function. Nat Med 2018; 24: 39-49.

41 Yuan Y, Han Q, Li S, et al. Wnt2b attenuates HSCs activation and liver fibrosis through negative regulating TLR4 signaling. Sci Rep 2017; 7: 3952. 